\title{
An intraductal approach to breast cancer risk assessment and screening in a diverse population L Bailey*, J Greif and J Mandelke
}

\author{
Address: Carol Ann Read Breast Health Center (CARBHC) of the Alta Bates Summit Medical Center, Oakland, CA, USA \\ Email: L Bailey* - baileylisa@msn.com \\ * Corresponding author
}

from 6th International Symposium on the Intraductal Approach to Breast Cancer

Santa Monica, CA, USA. 19-2I February 2009

Published: 24 July 2009

BMC Proceedings 2009, 3(Suppl 5):S24 doi:I0.I I86/I753-656I-3-S5-S24

This abstract is available from: http://www.biomedcentral.com/I753-656I/3/S5/S24

(c) 2009 Bailey et al; licensee BioMed Central Ltd.

The Gail Risk model was developed based on data from Caucasian women. Little information is available regarding breast cancer risk assessment in women of other ethnicities. Our study invited asymptomatic women of color presenting for screening mammography, to be interviewed with a risk questionnaire and to undergo aspiration of nipple fluid (NAF) for cytology. The HALO ${ }^{\mathrm{R}}$ system was used to aspirate the nipple fluid.

Thirty three patients participated in the study, including 24 African American, 7 Asian, and 2 Hispanic women all of whom completed the questionnaires. Twelve patients had NAF, and three of those patients had bilateral NAF aspirates, a total of 15 specimens. Ten of the twelve patients with NAF were African American, and all three of the patients with bilateral NAF were African American. The ages of the 33 patients in the study ranged from 40 to 72 years, with an average age of 53 , while the patients with NAF were ages 40 to 60 years with an average age of 51 years.

The age of menarche and prior use of oral contraceptives were similar in the NAF and Non-NAF groups. Only 7 of the 33 patients had a history of using any type of HRT. Only two patients had a history of fertility medications. No patients had DES exposure. Fourteen patients had a history of cigarette smoking, including 6 of the NAF patients and 8 of the non-NAF patients. Twelve patients had a family history of breast cancer, 5 with NAF and 7 without NAF, with a higher rate in the NAF group (71\%) vs. the non-NAF group (50\%). There were 20 patients who stated that they were postmenopausal, and 15 of these patients were in the non-NAF group.

No patients with NAF had abnormal cytology. Eleven of these samples had no ductal epithelial cells present, and the other 4 samples had rare or scant numbers of epithelial cells present. All of the samples had Thin Prep Concentration $^{R}$ used for slide preparation, but only three samples had adequate fluid available for cell block. Three patients (one with NAF and two without NAF) had abnormal mammograms and are having additional imaging.

We have shown that it is feasible to engage women of color in a study of breast cancer risk factors and aspiration of nipple fluid for cytology. Oakland, California is a very diverse community with multiple ethnic groups living in the city. One challenge with the Hispanic and Asian women was related to language barriers, and in a larger study a greater effort will be needed to be able to involve more patients from these communities. We were disappointed in the low number of patients with NAF samples and the adequacy of the samples, and are evaluating the issues that contributed to that problem. 